\title{
Limit-Point/Limit-Circle Results for Superlinear Damped Equations
}

\author{
M. Bartušek ${ }^{1}$ and John R. Graef ${ }^{2}$ \\ ${ }^{1}$ Faculty of Science, Masaryk University Brno, Kotlářská 2, 61137 Brno, Czech Republic \\ ${ }^{2}$ Department of Mathematics, University of Tennessee at Chattanooga, Chattanooga, TN 37403, USA
}

Correspondence should be addressed to John R. Graef; john-graef@utc.edu

Received 15 April 2013; Accepted 6 September 2013

Academic Editor: Allan Peterson

Copyright (C) 2013 M. Bartušek and J. R. Graef. This is an open access article distributed under the Creative Commons Attribution License, which permits unrestricted use, distribution, and reproduction in any medium, provided the original work is properly cited.

The authors study the nonlinear limit-point and limit-circle properties for second-order nonlinear damped differential equations of the form $\left(a(t)\left|y^{\prime}\right|^{p-1} y^{\prime}\right)^{\prime}+b(t)\left|y^{\prime}\right|^{q-1} y^{\prime}+r(t)|y|^{\lambda-1} y=0$, where $0<q \leq p \leq \lambda, a(t)>0$, and $r(t)>0$. Examples to illustrate the main results are included.

\section{Introduction}

In this paper, we continue the study of the nonlinear limit-point and limit-circle properties for the second-order damped equation

$$
\left(a(t)\left|y^{\prime}\right|^{p-1} y^{\prime}\right)^{\prime}+b(t)\left|y^{\prime}\right|^{q-1} y^{\prime}+r(t)|y|^{\lambda-1} y=0,
$$

where $\mathbb{R}_{+}=[0, \infty), \mathbb{R}=(-\infty, \infty), 0<q \leq p \leq \lambda, a \in$ $C^{1}\left(\mathbb{R}_{+}\right), b \in C^{0}\left(\mathbb{R}_{+}\right), r \in C^{1}\left(\mathbb{R}_{+}\right), a(t)>0$, and $r(t)>0$. We also consider the special case of (1) with $p=q$, namely,

$$
\left(a(t)\left|y^{\prime}\right|^{p-1} y^{\prime}\right)^{\prime}+b(t)\left|y^{\prime}\right|^{p-1} y^{\prime}+r(t)|y|^{\lambda-1} y=0 .
$$

Previous results of this type for damped equations can be found in the papers of Shao and Song [1], Xing et al. [2], and the present authors [3]. Later in this paper we will compare the results here with those previously known.

The limit-point/limit-circle problem has its origins in the work of Weyl [4] over 100 years ago. Weyl considered the second-order linear eigenvalue problem

$$
y^{\prime \prime}+r(t) y=\theta y, \quad \theta \in \mathbb{C}
$$

and classified this equation to be of the limit-circle type if every solution belongs to $L^{2}$, that is,

$$
\int_{0}^{\infty} y^{2}(\sigma) d \sigma<\infty
$$

and to be of the limit-point type if at least one solution $y(t)$ does not belong to $L^{2}$, that is,

$$
\int_{0}^{\infty} y^{2}(\sigma) d \sigma=\infty
$$

The limit-point/limit-circle problem then becomes that of determining conditions on the coefficient function $r$ that allows us to distinguish between these two cases. Weyl also proved that the linear equation $(\mathrm{C})$ always has at least one square integrable solution provided $\operatorname{Im} \theta \neq 0$. The problem then reduces to whether $(\mathrm{C})$ has one (limit-point case) or two (limit-circle case) square integrable solutions; this has come to be known as the Weyl Alternative. Weyl also showed that if (C) is limit-circle for some $\theta_{0} \in \mathbb{C}$, then it is limit-circle for all $\theta \in \mathbb{C}$. In particular, this is true for $\theta=0$, so that if we can show that the equation

$$
y^{\prime \prime}+r(t) y=0
$$

is limit-circle, then $(\mathrm{C})$ is limit-circle for all values of $\theta$, and if $(\mathrm{L})$ is not limit-circle, then $(\mathrm{C})$ is not limit-circle for any value of $\theta$. However, for $(\mathrm{L})$ we are not guaranteed that there is at least one square integrable solution.

In the years since Weyl's original work there has been a great deal of interest in this problem due to its relationship with the solution of certain boundary value problems. By comparison, the analogous problem for nonlinear equations 
is relatively new and has not been as extensively studied as the linear case.

In what follows, we will only consider solutions defined on their maximal interval of existence to the right. We next define what we mean by a proper solution.

Definition 1. A solution of (1) is said to be proper if it is defined on $\mathbb{R}_{+}$and is nontrivial in any neighborhood of $\infty$.

Remark 2. Under the covering assumptions here, the functions $a, b$, and $r$ are smooth enough so that all solutions of (1) are defined for large $t$ (see [5, Theorem 2(i)]). Moreover, all nontrivial solutions of (1) are proper if either $b \leq 0$ on $\mathbb{R}_{+}$or $q=p($ see $[5$, Theorem 4$])$.

The nonlinear limit-point/limit-circle problem originated in the work of Graef $[6,7]$ and Graef and Spikes [8]. The history and a survey of what is known about the linear and nonlinear problems as well as their relationships with other properties of solutions such as boundedness, oscillation, and convergence to zero, can be found in the monograph by Bartušek et al. [9] as well as the recent papers of Bartušek and Graef [10-14]. The nonlinear limit-point and limit-circle properties of solutions are defined as follows (see [9] and the papers $[3,6,7,10-18])$.

Definition 3. A solution $y$ of (1) is said to be of the nonlinear limit-circle type if

$$
\int_{0}^{\infty}|y(t)|^{\lambda+1} d t<\infty
$$

and it is said to be of the nonlinear limit-point type otherwise, that is, if

$$
\int_{0}^{\infty}|y(t)|^{\lambda+1} d t=\infty
$$

Equation (1) will be said to be of the nonlinear limit-circle type if every solution $y$ of (1) satisfies (NLC) and to be of the nonlinear limit-point type if there is at least one solution $y$ for which (NLP) holds.

We can write (1) as the equivalent system

$$
\begin{gathered}
y_{1}^{\prime}=a^{-1 / p}(t)\left|y_{2}\right|^{1 / p} \operatorname{sgn} y_{2}, \\
y_{2}^{\prime}=-b(t) a^{-q / p}(t)\left|y_{2}\right|^{q / p} \operatorname{sgn} y_{2}-r(t)\left|y_{1}\right|^{\lambda} \operatorname{sgn} y_{1},
\end{gathered}
$$

where the relationship between a solution $y$ of (1) and a solution $\left(y_{1}, y_{2}\right)$ of the system (5) is given by

$$
y_{1}(t)=y(t), \quad y_{2}(t)=a(t)\left|y^{\prime}(t)\right|^{p-1} y^{\prime}(t) .
$$

Also of interest here is what we call the strong nonlinear limit-point and strong nonlinear limit-circle properties of solutions of (1) as can be found in the following definitions. These notions were first introduced in $[17,18]$, respectively, and further studied, for example, in $[10,11]$. We define the function $R: \mathbb{R}_{+} \rightarrow \mathbb{R}$ by

$$
R(t)=a^{1 / p}(t) r(t)
$$

and the constant $\delta$ by

$$
\delta=\frac{p+1}{p}
$$

Definition 4. A solution $y$ of (1) is said to be of the strong nonlinear limit-point type if

$$
\int_{0}^{\infty}|y(t)|^{\lambda+1} d t=\infty, \quad \int_{0}^{\infty} \frac{\left|y_{2}(t)\right|^{\delta}}{R(t)} d t=\infty .
$$

Equation (1) is said to be of the strong nonlinear limit-point type if every proper solution is of the strong nonlinear limitpoint type and there is at least one proper solution.

Definition 5. A solution $y$ of (1) is said to be of the strong nonlinear limit-circle type if

$$
\int_{0}^{\infty}|y(t)|^{\lambda+1} d t<\infty, \quad \int_{0}^{\infty} \frac{\left|y_{2}(t)\right|^{\delta}}{R(t)} d t<\infty
$$

Equation (1) is said to be of the strong nonlinear limit-circle type if every solution is of the strong nonlinear limit-circle type.

Notice that if $b(t) \equiv 0,(1)$ reduces to

$$
\left(a(t)\left|y^{\prime}\right|^{p-1} y^{\prime}\right)^{\prime}+r(t)|y|^{\lambda-1} y=0
$$

and moreover, if $\lambda=p$, then (11) takes the form

$$
\left(a(t)\left|y^{\prime}\right|^{p-1} y^{\prime}\right)^{\prime}+r(t)|y|^{p-1} y=0
$$

This is the well-known half-linear equation, a general discussion of which can be found in the monograph by Došlý and Rehák [19]. Using the terminology introduced by the authors in $[3,11,16]$, if $\lambda>p$, we say that (1) or (11) is of the super-halflinear type, and if $\lambda<p$, we will say that it is of the sub-halflinear type. Since in this paper we are assuming that $\lambda \geq p$, we are in the half-linear and super-half-linear cases. In [3], we considered the sub-half-linear case of (1) and (11).

The limit-point/limit-circle problem for the damped equation

$$
\left(a(t) y^{\prime}\right)^{\prime}+b(t) y^{\prime}+r(t) y^{\lambda}=0
$$

with $b(t) \geq 0$ was studied in [1] with $\lambda \leq 1$ being the ratio of odd positive integers and in [2] with $\lambda \geq 1$ being an odd integer. The results in both of these papers tend to be modifications of results in [6-8] to accommodate the damping term. We will say more about the relationship between the results in $[1,2]$ and the present paper in Section 5. 
It will be convenient to define the following constants:

$$
\begin{gathered}
\alpha=\frac{p+1}{(\lambda+2) p+1}, \quad \beta=\frac{(\lambda+1) p}{(\lambda+2) p+1}, \\
\gamma=\frac{p+1}{p(\lambda+1)}, \quad \alpha_{1}=\alpha \gamma^{-1 /(\lambda+1)}, \\
\beta_{1}=\frac{p}{(\lambda+2) p+1}, \\
\beta_{2}=\frac{(\lambda+1)(p+1)}{\lambda(p-q)+(\lambda-q)}
\end{gathered}
$$

for either $p>q$ or $\lambda>q$,

$$
\begin{gathered}
\omega=\frac{1}{\lambda+1}+\frac{p}{p+1}, \quad \omega_{1}=\frac{q+1}{p+1}, \\
\omega_{2}=\frac{1}{\lambda+1}+\frac{q}{p+1}, \\
v=\frac{q}{p} \leq 1, \quad v_{1}=\frac{q-p}{(\lambda+2) p+1} .
\end{gathered}
$$

Notice that $\alpha=1-\beta, \omega_{2} \leq \omega_{1}$, and $\omega \leq 1$. We define the function $g: \mathbb{R}_{+} \rightarrow \mathbb{R}$ by

$$
g(t)=-\frac{a^{1 / p}(t) R^{\prime}(t)}{R^{\alpha+1}(t)},
$$

and sometimes we will make use of the assumption that

$$
\lim _{t \rightarrow \infty} g(t)=0, \quad \int_{0}^{\infty}\left|g^{\prime}(s)\right| d s<\infty .
$$

If (16) holds, we define the constants

$$
\gamma_{1}=\alpha \gamma^{-1 /(\lambda+1)} \sup _{s \in \mathbb{R}_{+}}|g(s)|, \quad \gamma_{2}=\delta+\gamma_{1} .
$$

For any solution $y: \mathbb{R}_{+} \rightarrow \mathbb{R}$ of $(1)$, we let

$$
\begin{aligned}
F(t) & =R^{\beta}(t)\left[\frac{a(t)}{r(t)}\left|y^{\prime}(t)\right|^{p+1}+\gamma|y(t)|^{\lambda+1}\right] \\
& =R^{\beta}(t)\left(\frac{\left|y_{2}(t)\right|^{\delta}}{R(t)}+\gamma|y(t)|^{\lambda+1}\right) .
\end{aligned}
$$

Note that $F \geq 0$ on $\mathbb{R}_{+}$for every solution of (1).

For any continuous function $h: \mathbb{R}_{+} \rightarrow \mathbb{R}$, we let $h_{+}(t)=$ $\max \{h(t), 0\}$ and $h_{-}(t)=\max \{-h(t), 0\}$ so that $h(t)=h_{+}(t)-$ $h_{-}(t)$.

In Section 2, we give some preliminary lemmas. Section 3 contains our main results on (1), and in Section 4 we study (2). Examples to illustrate our results and to compare our results to previously known ones are given in Section 5.

\section{Lemmas}

In this section we establish some lemmas that will be needed to prove the main results in this paper.
Lemma 1. Let either $p=q$ or $b \leq 0$ on $\mathbb{R}_{+}$. Then for every nontrivial solution $y$ of (1) one has $F(t)>0$ for large $t$.

Proof. Let $y$ be a nontrivial solution of (1). Then (18) implies the existence of $T \geq 0$ such that $F(T)>0$. Suppose, to the contrary, that $F\left(t_{0}\right)=0$ for some $t_{0}>T$. Then (18) implies $y\left(t_{0}\right)=y^{\prime}\left(t_{0}\right)=0$ and so (1) has the solution $\bar{y}$ defined by

$$
\begin{array}{cc}
\bar{y}(t)=y(t) \quad \text { for } t \in\left[0, t_{0}\right], \\
\bar{y}(t)=0 \quad \text { for } t \geq t_{0} .
\end{array}
$$

But this contradicts Remark 2 and proves the lemma.

Lemma 2. Let $y$ be a solution of (1). Then

(i) for $t \in \mathbb{R}_{+}$, one has

$$
\begin{gathered}
|y(t)| \leq \gamma^{-1 /(\lambda+1)} R^{-\beta_{1}}(t) F^{1 /(\lambda+1)}(t), \\
\left|y_{2}(t)\right| \leq R^{\beta_{1}}(t) F^{p /(p+1)}(t) ;
\end{gathered}
$$

(ii) for $0 \leq \tau<t$, one has

$$
\begin{gathered}
F(t)=F(\tau)-\alpha g(\tau) y(\tau) y_{2}(\tau)+\alpha g(t) y(t) y_{2}(t) \\
-\alpha \int_{\tau}^{t} g^{\prime}(s) y(s) y_{2}(s) d s \\
-\int_{\tau}^{t}\left[\delta R^{-\alpha}(s)\left|y_{2}(s)\right|^{1 / p} \operatorname{sgn} y_{2}(s)\right. \\
\quad-\alpha g(s) y(s)] \frac{b(s)}{a^{v}(s)}\left|y_{2}(s)\right|^{\nu-1} y_{2}(s) d s, \\
\mid \int_{\tau}^{t}\left[\delta R^{-\alpha}(s)\left|y_{2}(s)\right|^{1 / p} \operatorname{sgn} y_{2}(s)-\alpha g(s) y(s)\right] \\
\times \frac{b(s)}{a^{v}(s)}\left|y_{2}(s)\right|^{\nu-1} y_{2}(s) d s \mid \\
\leq \delta \int_{\tau}^{t} \frac{|b(s)|}{a^{v}(s)} R^{v_{1}}(s) F^{\omega_{1}}(s) d s \\
+\gamma_{1} \int_{\tau}^{t} \frac{|b(s)|}{a^{v}(s)} R^{v_{1}}(s) F^{\omega_{2}}(s) d s .
\end{gathered}
$$

Proof. Let $y$ be a solution of (1). Then it is a solution of the equation

$$
\left(a(t)\left|z^{\prime}\right|^{p-1} z^{\prime}\right)^{\prime}+r(t)|z|^{\lambda-1} z=e(t)
$$

with $e(t)$

$$
-b(t)\left|y^{\prime}(t)\right|^{q-1} y^{\prime}(t)
$$
$-\left(b(t) / a^{v}(t)\right)\left|y_{2}(t)\right|^{v-1} y_{2}(t)$. The expressions (20) and (21) follow from Lemma 1.2 in [16] applied to (23). Inequality (22) follows from (20).

The next two lemmas give us sufficient conditions for the boundedness of $F$ from above and from below by positive constants. 
Lemma 3. In addition to (16), assume that

$$
\int_{0}^{\infty} \frac{|b(t)|}{a^{v}(t)} R^{v_{1}}(t) d t<\infty
$$

and one of the following conditions holds:

(i) $\lambda=p=q$;

(ii) $b \leq 0$ for large $t$ and

$$
\begin{gathered}
\liminf _{t \rightarrow \infty} R^{-\beta}(t)\left\{\int_{t}^{\infty}\left[\left|g^{\prime}(s)\right|+\frac{|b(s)|}{a^{v}(s)} R^{v_{1}}(s)\right] d s\right\}^{\beta_{2}} \\
\times \exp \left\{\int_{0}^{t} \frac{R_{+}^{\prime}(s)}{R(s)} d s\right\}=0 ;
\end{gathered}
$$

or

(iii) $p=q<\lambda$ and (25) holds.

Then for any nontrivial solution $y$ of (1) defined on $\mathbb{R}_{+}$, the function $F$ is bounded from below for large $t$ by a positive constant depending on $y$.

Proof. Suppose, to the contrary, that there is a nontrivial solution of (1) such that

$$
\liminf _{t \rightarrow \infty} F(t)=0
$$

By Lemma $1, F(t)>0$ for large $t$. Let $\bar{t} \in \mathbb{R}_{+}$be such that $F(t)>0$ for $t \geq \bar{t}$ and

$$
\begin{gathered}
2 \alpha_{1} \sup _{s \in[\bar{t}, \infty)}|g(s)|+\alpha_{1} \int_{\bar{t}}^{\infty}\left|g^{\prime}(s)\right| d s \\
\quad+\gamma_{2} \int_{\bar{t}}^{\infty} \frac{|b(s)|}{a^{v}(s)} R^{v_{1}}(s) d s \leq \frac{1}{4}
\end{gathered}
$$

the existence of such a $\bar{t}$ follows from (16) and (24). Then, for any $t_{0} \geq \bar{t}$ such that $F\left(t_{0}\right) \leq 1$, there exist $\tau$ and $\sigma$ such that $t_{0} \leq \sigma<\tau$ and

$$
2 F(\tau)=F(\sigma)=F\left(t_{0}\right)>0, \quad F(\tau) \leq F(t) \leq F(\sigma)
$$

for $\sigma \leq t \leq \tau$. Then (20) implies

$$
\left|y(t) y_{2}(t)\right| \leq \gamma^{-1 /(\lambda+1)} F^{\omega}(t)
$$

on $\mathbb{R}_{+}$. From (21) (with $\tau=\sigma$ and $t=\tau$ ), inequalities (22) and (29), and the fact that $F(\sigma) \leq 1$, we have

$$
\begin{aligned}
\frac{F(\sigma)}{2}= & F(\sigma)-F(\tau) \\
\leq & {\left[\alpha_{1}|g(\tau)|+\alpha_{1}|g(\sigma)|+\alpha_{1} \int_{\sigma}^{\infty}\left|g^{\prime}(s)\right| d s\right] F^{\omega}(\sigma) } \\
& +\int_{\sigma}^{\infty} \frac{|b(s)|}{a^{v}(s)} R^{v_{1}}(s) d s\left(\gamma_{1} F^{\omega_{2}}(\sigma)+\delta F^{\omega_{1}}(\sigma)\right)
\end{aligned}
$$

Since $\min \left(\omega, \omega_{1}, \omega_{2}\right)=\omega_{2} \leq 1,(27)$ and (30) imply

$$
F(\sigma) \leq \frac{1}{2} F^{\omega_{2}}(\sigma)
$$

It is easy to see that $\omega_{2}=1$ if and only if $p=\lambda=q$.

In this case (31) and $F>0$ give us a contradiction and the statement of the lemma holds in case (i).

Suppose that $p=\lambda=q$ does not hold; this implies $\omega_{2} \neq 1$. Since $g$ is of bounded variation and $\lim _{t \rightarrow \infty} g(t)=0$, we see that

$$
|g(\sigma)|=|g(\sigma)-g(\infty)| \leq \int_{\sigma}^{\infty}\left|g^{\prime}(s)\right| d s .
$$

From this, (28), and (30), we obtain

$$
\begin{aligned}
F(\sigma) \leq 2\{3 & \alpha_{1} \int_{\sigma}^{\infty}\left|g^{\prime}(s)\right| d s \\
& \left.+\gamma_{2} \int_{\sigma}^{\infty} \frac{|b(s)|}{a^{v}(s)} R^{v_{1}}(s) d s\right\} F^{\omega_{2}}(\sigma),
\end{aligned}
$$

or

$$
\begin{aligned}
F\left(t_{0}\right) & =F(\sigma) \\
& \leq K\left\{\int_{\sigma}^{\infty}\left[\left|g^{\prime}(s)\right|+\frac{|b(s)|}{a^{v}(s)} R^{v_{1}}(s)\right] d s\right\}^{\beta_{2}}
\end{aligned}
$$

with $K=\left(6 \alpha_{1}+2 \gamma_{2}\right)^{\beta_{2}}$. Hence,

$$
F(t) \leq K\left\{\int_{t}^{\infty}\left[\left|g^{\prime}(s)\right|+\frac{|b(s)|}{a^{v}(s)} R^{v_{1}}(s)\right] d s\right\}^{\beta_{2}}
$$

for all $t \geq \bar{t}$ such that $F(t) \leq 1$. At the same time, (31) implies $F(t) \leq 2^{-\beta_{2}}<1$ for these values of $t$. Thus, (35) holds for all $t \geq \bar{t}$ and so $\lim _{t \rightarrow \infty} F(t)=0$.

Now in cases (ii) and (iii) we can actually estimate a bound from below on $F$. Let

$$
Z(t)=F(t) R^{-\beta}(t) .
$$

Then (18) and (36) imply

$$
Z^{\prime}(t)=\left(R^{-1}(t)\right)^{\prime}\left|y_{2}(t)\right|^{\delta}-\delta r^{-1}(t) b(t)\left|y^{\prime}(t)\right|^{q+1} .
$$

Define

$$
S(t)= \begin{cases}-\left(R^{-1}(t)\right)_{-}^{\prime}, & \text { in case (ii) } \\ -\left(R^{-1}(t)\right)_{-}^{\prime}-\delta \frac{b_{+}(t)}{r(t)} a^{-\delta}(t), & \text { in case (iii) }\end{cases}
$$

Suppose case (ii) holds. Then (36) and (37) imply

$$
Z^{\prime}(t) \geq S(t) R(t) Z(t) .
$$

Suppose case (iii) holds. Then (36) and (37) imply

$$
\begin{aligned}
Z^{\prime}(t) & \geq-\left(R^{-1}(t)\right)_{-}^{\prime}\left|y_{2}(t)\right|^{\delta}-\delta \frac{b_{+}(t)}{r(t)} a^{-\delta}(t)\left|y_{2}(t)\right|^{\delta} \\
& \geq S(t) R(t) Z(t) .
\end{aligned}
$$


Hence, (39) holds in both cases (ii) and (iii) for $t \geq \bar{t}$. So

$$
Z(t) \geq Z(\bar{t}) \exp \left\{\int_{\bar{t}}^{t} S(\sigma) R(\sigma) d \sigma\right\},
$$

and in view of (35) and the fact that $S(t) \leq 0$,

$$
\begin{aligned}
& Z(\bar{t}) R^{\beta}(t) \exp \left\{\int_{\bar{t}}^{t} S(\sigma) R(\sigma) d \sigma\right\} \\
& \quad \leq F(t) \leq K\left\{\int_{t}^{\infty}\left[\left|g^{\prime}(s)\right|+\frac{|b(s)|}{a^{v}(s)} R^{v_{1}}(s)\right] d s\right\}^{\beta_{2}},
\end{aligned}
$$

which contradicts (25). Notice that

$$
\exp \left\{-\int_{\bar{t}}^{t} S(\sigma) R(\sigma) d \sigma\right\} \leq C \exp \int_{0}^{t} \frac{R_{+}^{\prime}(s)}{R(s)} d s
$$

with $C=1$ in case (ii) and $C=\exp \left\{\delta \int_{0}^{\infty}(|b(s)| / a(s)) d s\right\}$ in case (iii).

Lemma 4. Let (16) and (24) hold. Then for every solution of (1), the function $F$ is bounded.

Proof. Let $y$ be a solution of (1) and let $\bar{t} \geq 0$ be such that (27) holds. Suppose that $F$ is not bounded, that is,

$$
\limsup _{t \rightarrow \infty} F(t)=\infty
$$

Then for any $t_{0} \geq \bar{t}$ with $F\left(t_{0}\right) \geq 1$, there exist $\sigma$ and $\tau$ such that $t_{0} \leq \sigma<\tau, F(\tau) / 2=F(\sigma)=F\left(t_{0}\right)$, and

$$
1 \leq F(\sigma) \leq F(t) \leq F(\tau) \quad \text { for } \sigma \leq t \leq \tau
$$

Setting $\tau=\sigma$ and $t=\tau$ in (20)-(22), we have (29) and

$$
\begin{aligned}
\frac{F(\tau)}{2}= & F(\tau)-F(\sigma) \\
\leq & {\left[\alpha_{1}|g(\sigma)|+\alpha_{1}|g(\tau)|+\alpha_{1} \int_{\sigma}^{\tau}\left|g^{\prime}(s)\right| d s\right] F^{\omega}(\tau) } \\
& +\int_{\sigma}^{\infty} \frac{|b(s)|}{a^{v}(s)} R^{v_{1}}(s) d s\left(\gamma_{1} F^{\omega_{2}}(\tau)+\delta F^{\omega_{1}}(\tau)\right)
\end{aligned}
$$

Since $\max \left(\omega_{1}, \omega_{2}, \omega_{3}\right) \leq 1,(27)$ and (45) imply

$$
\begin{aligned}
F(\tau) \leq 2\left[2 \alpha_{1} \max _{s \in[\bar{t}, \infty)}|g(s)|+\alpha_{1} \int_{\bar{t}}^{\infty}\left|g^{\prime}(s)\right| d s\right. \\
\left.+\gamma_{2} \int_{\bar{t}}^{\infty} \frac{|b(s)|}{a^{v}(s)} R^{v_{1}}(s) d s\right] F(\tau) \leq \frac{F(\tau)}{2} .
\end{aligned}
$$

This contradiction proves that $F$ is bounded.

Lemma 5. Let (16) and (24) hold. Then there exists a solution $y$ of (1) and positive constants $c_{1}$ and $c_{2}$ and $t_{0} \geq 0$ such that

$$
0<c_{1} \leq F(t) \leq c_{2} \quad \text { for } t \in\left[t_{0}, \infty\right) \text {. }
$$

Proof. Assumption (16) implies that $g$ is bounded, so we can choose $t_{0} \in \mathbb{R}_{+}$such that

$$
\begin{gathered}
M=\frac{1}{4}\left[3 \alpha_{1}+\delta+\gamma_{1}\right]^{-1}, \\
|g(t)| \leq M \quad \text { for } t \geq t_{0}, \quad \int_{t_{0}}^{\infty}\left|g^{\prime}(s)\right| d s \leq M \\
\int_{t_{0}}^{\infty} \frac{|b(s)|}{a^{v}(s)} R^{v_{1}}(s) d s \leq M .
\end{gathered}
$$

Consider a solution $y$ of (1) such that $F\left(t_{0}\right)=1$. First, we will show that

$$
F(t) \leq \frac{3}{2} \quad \text { for } t \geq t_{0}
$$

Suppose (50) does not hold. Then there exist $t_{2}>t_{1} \geq t_{0}$ such that

$$
F\left(t_{2}\right)=\frac{3}{2}, \quad F\left(t_{1}\right)=1, \quad 1<F(t)<\frac{3}{2}
$$

for $t \in\left(t_{1}, t_{2}\right)$. Lemma 2 (with $\tau=t_{1}$ and $t=t_{2}$ ), together with the facts that $\omega \leq 1$ and $\omega_{2} \leq \omega_{1} \leq 1$, implies

$$
\begin{aligned}
\frac{1}{2} & =\frac{F\left(t_{1}\right)}{2}=F\left(t_{2}\right)-F\left(t_{1}\right) \\
& \leq 3 \alpha_{1} M F^{\omega}\left(t_{2}\right)+\delta M F^{\omega_{1}}\left(t_{2}\right)+\gamma_{1} M F^{\omega_{2}}\left(t_{2}\right) \\
& \leq \frac{3}{2} M\left[3 \alpha_{1}+\delta+\gamma_{1}\right]=\frac{3}{8} .
\end{aligned}
$$

This contradiction shows that (50) holds.

Now, Lemma 2 (with $t=t, \tau=t_{0}$ ) similarly implies

$$
\begin{aligned}
|F(t)-1| & \leq 3 \alpha_{1} M\left(\frac{3}{2}\right)^{\omega}+\delta M\left(\frac{3}{2}\right)^{\omega_{1}}+\gamma_{1} M\left(\frac{3}{2}\right)^{\omega_{2}} \\
& \leq \frac{3}{2} M\left[3 \alpha_{1}+\delta+\gamma_{1}\right]<\frac{1}{2}
\end{aligned}
$$

for $t \geq t_{0}$. From this and from (50) we have

$$
\frac{1}{2} \leq F(t) \leq \frac{3}{2} \text { for } t \geq t_{0} \text {. }
$$

Next, we prove that $y$ is defined on $\left[0, t_{0}\right]$. Suppose to the contrary that $y$ is defined on $\left(a, t_{0}\right)$ with $0 \leq a<t_{0}$ and $y$ cannot be extended to $t=a$. Then $\lim \sup _{t \rightarrow a^{+}}\left|y_{2}(t)\right|=\infty$. The change of variables $x=t_{0}-t$ and $y(t)=Y(x)$ transforms (1) into

$$
\begin{gathered}
\left(a\left(t_{0}-x\right)|\dot{Y}|^{p-1} \dot{Y}\right) \cdot b\left(t_{0}-x\right)|\dot{Y}|^{q-1} \dot{Y} \\
+r\left(t_{0}-x\right)|Y|^{\lambda-1} Y=0, \quad \cdots \cdots=\frac{d}{d s},
\end{gathered}
$$

with the noncontinuable solution $Y(x)$ defined on $\left[0, t_{0}-a\right)$ and $\lim \sup _{x \rightarrow\left(t_{0}-a\right)^{-}}|\dot{Y}(x)|=\infty$. This contradicts Remark 2 applied to (55) and proves that $y$ is defined on $\mathbb{R}_{+}$. The conclusion of the lemma then follows from (54). 
Lemma 6. Suppose that (16) and (24) hold and

$$
\int_{0}^{\infty} R^{-\beta}(t) d t=\infty
$$

In addition, assume that either

$$
\lim _{t \rightarrow \infty} R^{\beta}(t)\left[\left|\left(\frac{1}{r(t)}\right)^{\prime}\right|+\frac{|b(t)|}{a^{v}(t) r(t)} R^{v_{1}}(t)\right]=0
$$

or

$$
\lim _{t \rightarrow \infty} R^{\beta}(t)\left[\frac{\left|a^{\prime}(t)\right|}{a(t) r(t)}+\frac{|b(t)|}{a^{v}(t) r(t)} R^{v_{1}}(t)\right]=0
$$

holds. If $y$ is a solution of (1) with

$$
c_{1} \leq F(t) \leq c_{2}
$$

for larget and some positive constants $c_{1}$ and $c_{2}$, then

$$
\int_{0}^{\infty}|y(t)|^{\lambda+1} d t=\infty
$$

Moreover, if

$$
\limsup _{t \rightarrow \infty} r(t) \int_{0}^{t} R^{-\beta}(s) d s=\infty,
$$

then

$$
\int_{0}^{\infty} \frac{\left|y_{2}(t)\right|^{\delta}}{R(t)} d t=\infty
$$

That is, $y$ is of the strong nonlinear limit-point type.

Proof. Let $y$ be a nontrivial solution of (1) satisfying (59) on $[T, \infty) \subset \mathbb{R}_{+}$. Then, (20) and (59) imply the existence of $M_{1}$ such that

$$
\begin{gathered}
\left|y(t) y_{2}(t)\right| \leq \gamma^{-1 /(\lambda+1)} c_{2}^{\omega} \leq M_{1}, \\
|y(t)|\left|y_{2}(t)\right|^{v} \leq \gamma^{-1 /(\lambda+1)} c_{2}^{\omega_{2}} R^{v_{1}}(t) \leq M_{1} R^{v_{1}}(t)
\end{gathered}
$$

for $t \geq T$.

Let $t_{0} \geq T$ be such that

$$
|g(t)| \leq c_{1} \min (1, \gamma)\left(4 M_{1}\right)^{-1}
$$

holds for $t \geq t_{0}$. It follows from (5) that

$$
\begin{aligned}
\int_{t_{0}}^{t}|y(s)|^{\lambda+1} d s= & -\int_{t_{0}}^{t} \frac{y(s) y_{2}^{\prime}(s)}{r(s)} d s \\
& -\int_{t_{0}}^{t} \frac{b(s)}{a^{v}(s) r(s)} y(s)\left|y_{2}(s)\right|^{v} \operatorname{sgn} y_{2}(s) d s \\
= & -\frac{y(t) y_{2}(t)}{r(t)}+D+\int_{t_{0}}^{t} \frac{\left|y_{2}(s)\right|^{\delta}}{R(s)} d s+J(t),
\end{aligned}
$$

where $D=y\left(t_{0}\right) y_{2}\left(t_{0}\right) r^{-1}\left(t_{0}\right)$ and

$$
\begin{aligned}
J(t)=\int_{t_{0}}^{t} & {\left[\left(\frac{1}{r(s)}\right)^{\prime} y(s) y_{2}(s)\right.} \\
& \left.\quad-\frac{b(s)}{a^{v}(s) r(s)} y(s)\left|y_{2}(s)\right|^{v} \operatorname{sgn} y_{2}(s)\right] d s .
\end{aligned}
$$

From (63) and (66)

$$
|J(t)| \leq M_{1} \int_{t_{0}}^{t}\left[\left|\left(\frac{1}{r(s)}\right)^{\prime}\right|+\frac{|b(s)|}{a^{v}(s) r(s)} R^{v_{1}}(s)\right] d s .
$$

Moreover, (18) and (59) imply

$$
c_{3} \int_{t_{0}}^{t} R^{-\beta}(s) d s \leq \int_{t_{0}}^{t}|y(s)|^{\lambda+1} d s+\int_{t_{0}}^{t} \frac{\left|y_{2}(s)\right|^{\delta}}{R(s)} d s
$$

for $t \geq t_{0}$ with $c_{3}=c_{1} \min (1, \gamma)$.

Suppose (57) holds. By l'Hôspital's Rule, there exists $t_{1}>$ $t_{0}$ such that

$$
\begin{gathered}
\int_{t_{0}}^{t}\left[\left|\left(\frac{1}{r(s)}\right)^{\prime}\right|+\frac{|b(s)|}{a^{v}(s) r(s)} R^{v_{1}}(s)\right] d s \\
\leq \frac{c_{3}}{2 M_{1}} \int_{t_{0}}^{t} R^{-\beta}(s) d s
\end{gathered}
$$

for $t \geq t_{1}$. From this, (65) and (67), we have

$$
\begin{aligned}
& \left.\left|\int_{t_{0}}^{t}\right| y(s)\right|^{\lambda+1} d s-\int_{t_{0}}^{t} \frac{\left|y_{2}(s)\right|^{\delta}}{R(s)} d s+\frac{y(t) y_{2}(t)}{r(t)} \mid \\
& \quad \leq|D|+\frac{c_{3}}{2} \int_{t_{0}}^{t} R^{-\beta}(s) d s
\end{aligned}
$$

for $t \geq t_{1}$.

Now let (58) hold. Applying l'Hôspital's Rule, there exists $t_{1}>t_{0}$ such that

$$
\begin{gathered}
\int_{t_{0}}^{t}\left(\frac{\left|a^{\prime}(s)\right|}{p a(s) r(s)}+\frac{|b(s)|}{a^{v}(s) r(s)} R^{v_{1}}(s)\right) d s \\
\leq \frac{c_{3}}{4 M_{1}} \int_{t_{0}}^{t} R^{-\beta}(s) d s
\end{gathered}
$$

for $t \geq t_{1}$. Then using (64), (71), and the fact that $1 / r=$ $a^{1 / p} / R$, we have

$$
\begin{aligned}
\int_{t_{0}}^{t} & {\left[\left|\left(\frac{1}{r(s)}\right)^{\prime}\right|+\frac{|b(s)|}{a^{v}(s) r(s)} R^{v_{1}}(s)\right] d s } \\
& \leq \int_{t_{0}}^{t}\left(\frac{\left|a^{\prime}(s)\right|}{p a(s) r(s)}+\frac{|b(s)|}{a^{v}(s) r(s)} R^{v_{1}}(s)\right) d s+\int_{t_{0}}^{t} \frac{|g(s)|}{R^{\beta}(s)} d s \\
& \leq \frac{c_{3}}{2 M_{1}} \int_{t_{0}}^{t} R^{-\beta}(s) d s
\end{aligned}
$$


for $t \geq t_{1}$. This together with (65) and (67) implies that inequality (70) again holds. Thus, (70) holds if either (57) or (58) does. Moreover,

$$
\begin{aligned}
-|D| & -\frac{c_{3}}{2} \int_{t_{0}}^{t} R^{-\beta}(s) d s \\
& \leq \int_{t_{0}}^{t}|y(s)|^{\lambda+1} d s-\int_{t_{0}}^{t} \frac{\left|y_{2}(s)\right|^{\delta}}{R(s)} d s+\frac{y_{1}(t) y_{2}(t)}{r(t)} \\
& \leq|D|+\frac{c_{3}}{2} \int_{t_{0}}^{t} R^{-\beta}(s) d s
\end{aligned}
$$

for $t \geq t_{1}$. Adding (68) and the left-hand inequality in (73) gives

$$
\begin{aligned}
2 \int_{t_{0}}^{t}|y(s)|^{\lambda+1} d s+\frac{y(t) y_{2}(t)}{r(t)} & \\
& \geq \frac{c_{3}}{2} \int_{t_{0}}^{t} R^{-\beta}(s) d s-|D| \longrightarrow \infty
\end{aligned}
$$

as $t \rightarrow \infty$, while adding (68) and the right-hand inequality in (73) gives

$$
\begin{aligned}
2 \int_{t_{0}}^{t} \frac{\left|y_{2}(s)\right|^{\delta}}{R(s)} d s-\frac{y(t) y_{2}(t)}{r(t)} & \\
& \geq \frac{c_{3}}{2} \int_{t_{0}}^{t} R^{-\beta}(s) d s-|D| \longrightarrow \infty
\end{aligned}
$$

as $t \rightarrow \infty$.

If $y^{\prime}$ is oscillatory, let $\left\{t_{k}\right\}_{k=1}^{\infty} \rightarrow \infty$ be a sequence of zeros of $y^{\prime}$. Then letting $t=t_{k}$ in (74) and (75), we see that $y$ is a strong nonlinear limit-point type solution of (1).

If $y^{\prime}$ is nonoscillatory, then either

$$
y(t) y_{2}(t)>0
$$

or

$$
y(t) y_{2}(t)<0
$$

for large $t$. First we show (60) holds. Clearly (60) holds if (76) does, so suppose (77) holds. Then $y(t) y_{2}(t) r^{-1}(t)<0$ for large $t$ and (60) follows from (74).

Finally, assume (61) holds. From (61) and (63), it follows that there is a $t_{2} \geq t_{1}$ such that

$$
\left|\frac{y(t) y_{2}(t)}{r(t)}\right| \leq \frac{M_{1}}{r(t)} \leq \frac{c_{3}}{4} \int_{0}^{t} R^{-\beta}(s) d s
$$

for $t \geq t_{2}$; hence, (62) follows from this and from (75).

Remark 6. Lemma 6 actually holds for all positive $p, \lambda$, and $q$ regardless of their relative size.

\section{LP/LC Problem for (1)}

In this section we present our main results for (1).
Theorem 7. Assume that (16) and (24) hold. Then (1) is of the strong nonlinear limit-circle type if and only if

$$
\int_{0}^{\infty} R^{-\beta}(t) d t<\infty
$$

Proof. Suppose (79) holds and let $y$ be any nontrivial solution of (1) defined on $\mathbb{R}_{+}$. Then, by Lemma 4 , there is a positive constant $c$ such that $0 \leq F(t) \leq c$. Hence, from this and (18),

$$
\begin{aligned}
0 & \leq \gamma \int_{0}^{\infty}|y(t)|^{\lambda+1} d t+\int_{0}^{\infty} \frac{\left|y_{2}(t)\right|^{\delta}}{R(t)} d t \\
& =\int_{0}^{\infty} F(t) R^{-\beta}(t) d t \leq c \int_{0}^{\infty} R^{-\beta}(t) d t<\infty,
\end{aligned}
$$

so $y$ is of the strong nonlinear limit-circle type. Thus, (1) is of the strong nonlinear limit-circle type.

Now suppose that (79) does not hold, that is,

$$
\int_{0}^{\infty} R^{-\beta}(t) d t=\infty
$$

Let $y$ be a solution of (1) given by Lemma 5 . Then there is $c_{1}>0$ such that $c_{1} \leq F(t)$ on $\left[t_{0}, \infty\right) \subset \mathbb{R}_{+}$. Hence, from this and (18),

$$
\begin{aligned}
\gamma \int_{t_{0}}^{\infty} & |y(t)|^{\lambda+1} d t+\int_{t_{0}}^{\infty} \frac{\left|y_{2}(t)\right|^{\delta}}{R(t)} d t \\
& =\int_{t_{0}}^{\infty} F(t) R^{-\beta}(t) d t \geq c_{1} \int_{t_{0}}^{\infty} R^{-\beta}(t) d t=\infty
\end{aligned}
$$

Thus, either

$$
\int_{0}^{\infty}|y(t)|^{\lambda+1} d t=\infty \quad \text { or } \quad \int_{0}^{\infty} \frac{\left|y_{2}(t)\right|^{\delta}}{R(t)} d t=\infty,
$$

and so $y$ and (1) are not of the strong nonlinear limit-circle type.

Theorem 8. Let (16), (24), and either (57) or (58) hold. If

$$
\int_{0}^{\infty} R^{-\beta}(t) d t=\infty
$$

then (1) is of the nonlinear limit-point type.

Proof. The hypotheses of Lemmas 5 and 6 are satisfied; so if $y$ is a solution given by Lemma 5, then (60) holds and the conclusion follows.

Theorem 9. Let conditions (16), (24), and either (57) or (58) hold. Assume, in addition, that either (i) $\lambda=p=q$; (ii) $b \leq 0$ for large $t$ and (25) holds; or (iii) $p=q<\lambda$ and (25) holds. If (84) holds, then every nontrivial solution of (1) is of the nonlinear limit-point type. If, moreover, (61) holds, then (1) is of the strong nonlinear limit-point type.

Proof. Note that the hypotheses of Lemmas 3-6 are satisfied. Let $y$ be a nontrivial solution of (1) defined on $\mathbb{R}_{+}$. Then by 
Lemmas 3 and 4 , there are positive constants $c_{1}$ and $c_{2}$ such that

$$
0<c_{1} \leq F(t) \leq c_{2} \quad \text { for large } t
$$

Thus, by Lemma 6, (60) holds; moreover, if (61) holds, then so does (62). The existence of a nontrivial solution of (1) is given by Lemma 5 . This proves the theorem.

Remark 10. If $R$ is nondecreasing for large $t$, then condition (25) becomes

$$
\liminf _{t \rightarrow \infty} R^{\alpha}(t)\left\{\int_{t}^{\infty}\left[\left|g^{\prime}(s)\right|+\frac{|b(s)|}{a^{v}(s)} R^{v_{1}}(s)\right] d s\right\}^{\beta_{2}}=0 .
$$

If $R$ is nonincreasing for large $t$, then (25) becomes

$$
\liminf _{t \rightarrow \infty} R^{-\beta}\left\{\int_{t}^{\infty}\left[\left|g^{\prime}(s)\right|+\frac{|b(s)|}{a^{v}(s)} R^{v_{1}}(s)\right] d s\right\}^{\beta_{2}}=0 .
$$

\section{LP/LC Problem for (2)}

One of the main assumptions is Section 3 is condition (24), which takes the form

$$
\int_{0}^{\infty} \frac{|b(t)|}{a(t)} d t<\infty
$$

for (2). It is possible to remove this condition when studying (2). The technique to accomplish this is contained in the following lemma; a direct computation proves it (or see [3, Lemma 7]).

Lemma 11. Equation (2) and the equation

$$
\left(\bar{a}(t)\left|y^{\prime}\right|^{p-1} y^{\prime}\right)^{\prime}+\bar{r}(t)|y|^{\lambda-1} y=0
$$

are equivalent where

$$
\begin{aligned}
& \bar{a}(t)=a(t) \exp \left\{\int_{0}^{t} \frac{b(s)}{a(s)} d s\right\}, \\
& \bar{r}(t)=r(t) \exp \left\{\int_{0}^{t} \frac{b(s)}{a(s)} d s\right\} .
\end{aligned}
$$

That is, every solution of (2) is a solution of (89) and vice versa.

Based on this lemma, we can obtain results for (2) by combining Lemma 11 with known results for (89), such as those in $[10-14,16,18]$. Here we only present a sample of the many possibilities.

Set

$$
\begin{aligned}
\bar{R}(t)=\bar{a}^{1 / p}(t) \bar{r}(t) & =R(t) \exp \left\{\delta \int_{0}^{t} \frac{b(s)}{a(s)} d s\right\}, \\
\bar{g}(t)=-\frac{\bar{a}^{1 / p}(t) \bar{R}^{\prime}(t)}{\bar{R}^{\alpha+1}(t)}= & -\frac{a^{1 / p}(t)}{R^{\alpha+1}(t)}\left[R^{\prime}(t)+\delta \frac{b(t)}{a(t)} R(t)\right] \\
& \times \exp \left\{\frac{\lambda-p}{(\lambda+2) p+1} \int_{0}^{t} \frac{b(s)}{a(s)} d s\right\} .
\end{aligned}
$$

Theorem 12. (i) Let $\lambda>p$ and

$$
\int_{0}^{\infty} \bar{R}^{-\beta}(t)\left(\int_{0}^{t}\left|\bar{g}^{\prime}(s)\right| d s+1\right)^{(\lambda+1)(p+1) /(\lambda-p)} d t<\infty .
$$

Then (2) is of the nonlinear limit-circle type.

(ii) Assume that

$$
\lim _{t \rightarrow \infty} \bar{g}(t)=0, \quad \int_{0}^{\infty}\left|\bar{g}^{\prime}(t)\right| d t<\infty .
$$

Then (2) is of the strong nonlinear limit-circle type if and only if

$$
\int_{0}^{\infty} \bar{R}^{-\beta}(t) d t<\infty
$$

Proof. (i) If $\bar{g}$ is not identically a constant on $\mathbb{R}_{+}$the result follows from Lemma 11 and from [13, Theorem 1] applied to (89). If $\bar{g} \equiv$ const. on $\mathbb{R}_{+}$, then the statement follows from Theorem 7 applied to (89) and Lemma 11. Part (ii) follows from Lemma 11 and Theorem 7 applied to (89).

The next result follows from Lemma 11 and Theorem 8 applied to (89).

Theorem 13. Let (93) hold and assume that either

$$
\begin{aligned}
& \lim _{t \rightarrow \infty} R^{\beta}(t)\left(\frac{\left|r^{\prime}(t)\right|}{r^{2}(t)}+\frac{|b(t)|}{a(t) r(t)}\right) \\
& \quad \times \exp \left\{\frac{\lambda-p}{(\lambda+2) p+1} \int_{0}^{t} \frac{b(s)}{a(s)} d s\right\}=0
\end{aligned}
$$

or

$$
\begin{aligned}
& \lim _{t \rightarrow \infty} R^{\beta}(t) \frac{\left|a^{\prime}(t)\right|+|b(t)|}{a(t) r(t)} \\
& \quad \times \exp \left\{\frac{\lambda-p}{(\lambda+2) p+1} \int_{0}^{t} \frac{b(s)}{a(s)} d s\right\}=0 .
\end{aligned}
$$

If

$$
\int_{0}^{\infty}(\bar{R}(t))^{-\beta} d t=\infty
$$

then (2) is of the nonlinear limit-point type. for (2).

Our final theorem is a strong nonlinear limit-point result

Theorem 14. Assume that (93), (97), and either (95) or (96) hold. In addition, assume that either (i) $\lambda=p$; (ii) $b \leq 0$ on $\mathbb{R}_{+}$and

$$
\begin{aligned}
& \liminf _{t \rightarrow \infty}(\bar{R}(t))^{-\beta}\left\{\int_{t}^{\infty}\left|\bar{g}^{\prime}(s)\right| d s\right\}^{(\lambda+1)(p+1) /(\lambda-p)} \\
& \quad \times \exp \left\{\frac{\bar{R}_{+}^{\prime}(s)}{\bar{R}(s)} d s\right\}=0
\end{aligned}
$$


or (iii) $\lambda>p$ and (98) holds. Then every nontrivial solution of (2) is of the nonlinear limit-point type. If, moreover,

$$
\limsup _{t \rightarrow \infty} \bar{r}(t) \int_{0}^{t}(\bar{R}(s))^{-\beta} d s=\infty,
$$

then (2) is of the strong nonlinear limit-point type.

Proof. This follows from Lemma 11 and Theorem 9 applied to (89).

\section{Examples}

In this section we present some examples to illustrate our results.

Example 1. Consider the equation

$$
y^{\prime \prime}+t^{s} y^{\prime}+t^{\sigma}|y|^{\lambda-1} y=0, \quad t \geq 1
$$

with $s \in \mathbb{R}$ and $\lambda \geq 1$. We have the following results.

(i) If $s<-1$ and $\sigma>(\lambda+3) /(\lambda+1)$, then (100) is of the strong nonlinear limit-circle type by Theorem 7 .

(ii) If $s<-1$ and $-(\lambda+3) / 2<\sigma \leq(\lambda+3) /(\lambda+1)$, then (100) is of the strong nonlinear limit-point type by Theorem 8 .

(iii) If $\lambda=1, s<-1$, and $-2<\sigma \leq 2$, then (100) is of the strong nonlinear limit-point type by Theorem 9(i).

(iv) If $\lambda>1, s<-1-\sigma(\lambda-1) /(\lambda+3)(\lambda+1)$, and $0<\sigma \leq$ $(\lambda+3) /(\lambda+1)$, then (100) is of the strong nonlinear limit-point type by Theorem 9(iii).

The following example will allow us to compare our results to those in [2].

Example 2. Again consider (100) with $s \in \mathbb{R}$ and $\lambda \geq 1$. The following results hold.

(i) Equation (100) is of the nonlinear limit-circle type if
(a) $s<-1$ and $\sigma>(\lambda+3) /(\lambda+1)$ (by Theorem 12(ii));
(b) $s=-1$ and $\sigma>-(\lambda-1) /(\lambda+1)$ (by Theorem 12(i) if $\lambda>1$ and by Theorem 12(ii) if $\lambda=1$ );
(c) $s>-1, \lambda>1$, and $\sigma>(\lambda+3) /(\lambda+1)$ (by Theorem 12(i));
(d) $s>-1, \lambda=1$ and $s<\sigma / 2$ (by Theorem 12(ii)).

(ii) Equation (100) is of the nonlinear limit-point type if

$$
\begin{aligned}
& \text { (e) } s<-1 \text { and }-(\lambda+3) /(\lambda+1)<\sigma \leq(\lambda+3) /(\lambda+1) \\
& \text { (by Theorem 13); } \\
& \text { (f) } s=-1 \text { and }-2<\sigma \leq-(\lambda-1) /(\lambda+1) \text { (by } \\
& \text { Theorem 13). }
\end{aligned}
$$

By [2, Corollary 2.1], (100) is of the nonlinear limit-circle type if either (i) $\lambda=1, \sigma>2$, and $s<0$, or (ii) $\lambda>1,(\lambda+3) /(\lambda+$ $1)<\sigma<2(\lambda+3) /(\lambda-1)$, and $s<-\sigma((\lambda-1) / 2(\lambda+3))$. This shows that, in the case of nonlinear limit-circle type results, the results in [2] are a special case of those in this paper.
Remark 15. Theorem 2.3 in [2] appears to show that (100) is of the nonlinear limit-point type if $-1<\sigma \leq(\lambda+3) /(\lambda+$ $1), s \leq-\sigma((\lambda-1) / 2(\lambda+3))$, and $s<(\sigma-1) / 2$. We have a contradiction to our case (b) in Example 2 above if $s=-1$, $\lambda=1$, and $\sigma=1$. The proof of Theorem 2.3 in [2] is incorrect; in their expression for $\dot{V}$ the term " $-p(t)[a(t) r(t)]^{\beta-2 \alpha} x^{2 k}(s)$ " is missing.

In our next example we have that $b(t)$ in (1) (or (2)) is negative.

Example 3. Consider the equation

$$
y^{\prime \prime}-t^{s} y^{\prime}+t^{\sigma}|y|^{\lambda-1} y=0, \quad t \geq 1
$$

with $s \in \mathbb{R}, \lambda \geq 1$. Calculations show the following.

(i) Equation (101) is of the nonlinear limit-circle type if

(a) $s<-1$ and $\sigma>(\lambda+3) /(\lambda+1)$ (by Theorem 7$)$;

(b) $s=-1$ and $\sigma>-(\lambda-1) /(\lambda+1)$ (by Theorem 12(ii)).

(ii) Equation (101) is of the nonlinear limit-point type if

(c) $s<-1$ and $-(\lambda+3) / 2<\sigma \leq(\lambda+3) /(\lambda+1)$ (by Theorem 8);

(d) $s=-1$ and $-2(\lambda+1) /(\lambda+3)<\sigma \leq-(\lambda-1) /(\lambda+$ 1) (by Theorem 13);

(e) $s>-1$ and $\lambda>1$ (by Theorem 13);

(f) $s>-1, \lambda=1$, and $\sigma>\max \{2 s, s\}$ (by Theorem 13).

\section{Acknowledgment}

This research is supported by Grant no. 201/11/0768 of the Grant Agency of the Czech Republic.

\section{References}

[1] J. Shao and W. Song, "Limit circle/limit point criteria for second-order sublinear differential equations with damping term," Abstract and Applied Analysis, vol. 2011, Article ID 803137, 12 pages, 2011.

[2] L. Xing, W. Song, Z. Zhang, and Q. Xu, "Limit circle/limit point criteria for second-order superlinear differential equations with a damping term," Journal of Applied Mathematics, vol. 2012, Article ID 361961, 11 pages, 2012.

[3] M. Bartušek and J. R. Graef, "Limit-point/limit-circle results for equations with damping," Abstract and Applied Analysis, vol. 2012, Article ID 979138, 19 pages, 2012.

[4] H. Weyl, "Über gewöhnliche Differentialgleichungen mit Singularitäten und die zugehörigen Entwicklungen willkürlicher Funktionen," Mathematische Annalen, vol. 68, no. 2, pp. 220 269, 1910.

[5] M. Bartušek and E. Pekárková, "On existence of proper solutions of quasilinear second order differential equations," Electronic Journal of Qualitative Theory of Differential Equations, no. 5, pp. 1-14, 2007. 
[6] J. R. Graef, "Limit circle criteria and related properties for nonlinear equations," Journal of Differential Equations, vol. 35, no. 3, pp. 319-338, 1980.

[7] J. R. Graef, "Limit circle type results for sublinear equations," Pacific Journal of Mathematics, vol. 104, no. 1, pp. 85-94, 1983.

[8] J. R. Graef and P. W. Spikes, "On the nonlinear limit-point/limitcircle problem," Nonlinear Analysis: Theory, Methods \& Applications, vol. 7, no. 8, pp. 851-871, 1983.

[9] M. Bartušek, Z. Došlá, and J. R. Graef, The Nonlinear LimitPoint/Limit-Circle Problem, Birkhäuser, Boston, Mass, USA, 2004.

[10] M. Bartušek and J. R. Graef, "Strong nonlinear limit-point/ limit-circle properties for forced Thomas-Fermi equations with p-Laplacian," Panamerican Mathematical Journal, vol. 18, no. 1, pp. 73-88, 2008.

[11] M. Bartušek and J. R. Graef, "The strong nonlinear limitpoint/limit-circle properties for super-half-linear equations," Panamerican Mathematical Journal, vol. 17, no. 1, pp. 25-38, 2007.

[12] M. Bartušek and J. R. Graef, "Asymptotic properties of solutions of a forced second order differential equation with $p$-Laplacian," Panamerican Mathematical Journal, vol. 16, no. 2, pp. 41-59, 2006.

[13] M. Bartušek and J. R. Graef, "The nonlinear limit-point/limitcircle problem for second order equations with $p$-Laplacian," Dynamic Systems and Applications, vol. 14, no. 3-4, pp. 431-446, 2005.

[14] M. Bartušek and J. R. Graef, "Some limit-point and limit-circle results for second order Emden-Fowler equations," Applicable Analysis, vol. 83, no. 5, pp. 461-476, 2004.

[15] M. Bartušek and J. R. Graef, "Nonlinear limit-point/limit-circle properties of solutions of second order differential equations with p-Laplacian," International Journal of Pure and Applied Mathematics, vol. 45, no. 4, pp. 501-518, 2008.

[16] M. Bartušek and J. R. Graef, "Asymptotic behaviour of solutions of a differential equation with $p$-Laplacian and a forcing term," Differential Equations and Dynamical Systems, vol. 15, no. 1-2, pp. 61-87, 2007.

[17] M. Bartušek and J. R. Graef, "The strong nonlinear limit-point/ limit-circle properties for sub-half-linear equations," Dynamic Systems and Applications, vol. 15, no. 3-4, pp. 585-601, 2006.

[18] M. Bartušek and J. R. Graef, “The strong limit-point property for Emden-Fowler equations," Differential Equations and Dynamical Systems, vol. 14, no. 3-4, pp. 383-405, 2006.

[19] O. Došlý and P. Rehák, Half-Linear Differential Equations, vol. 202 of North-Holland Mathematics Studies, Elsevier, Amsterdam, The Netherlands, 2005. 


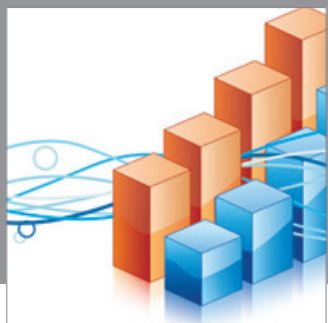

Advances in

Operations Research

mansans

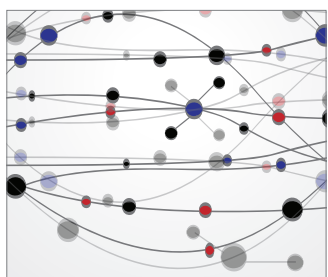

The Scientific World Journal
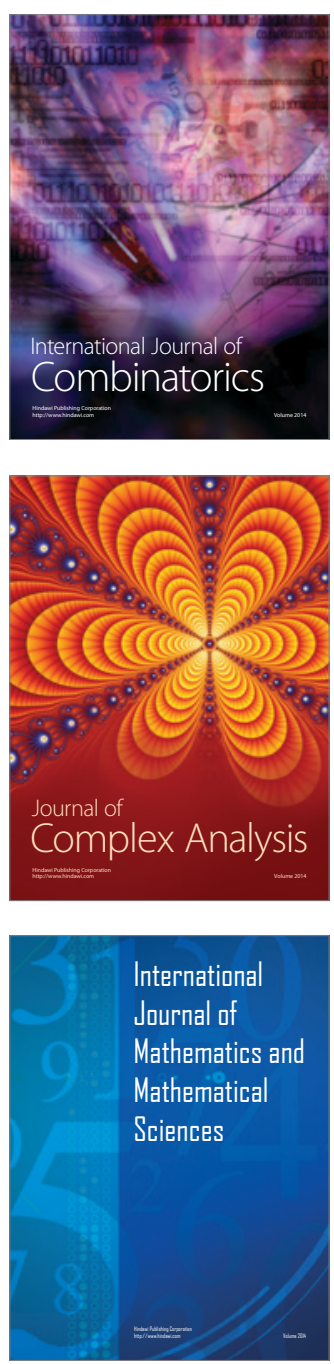
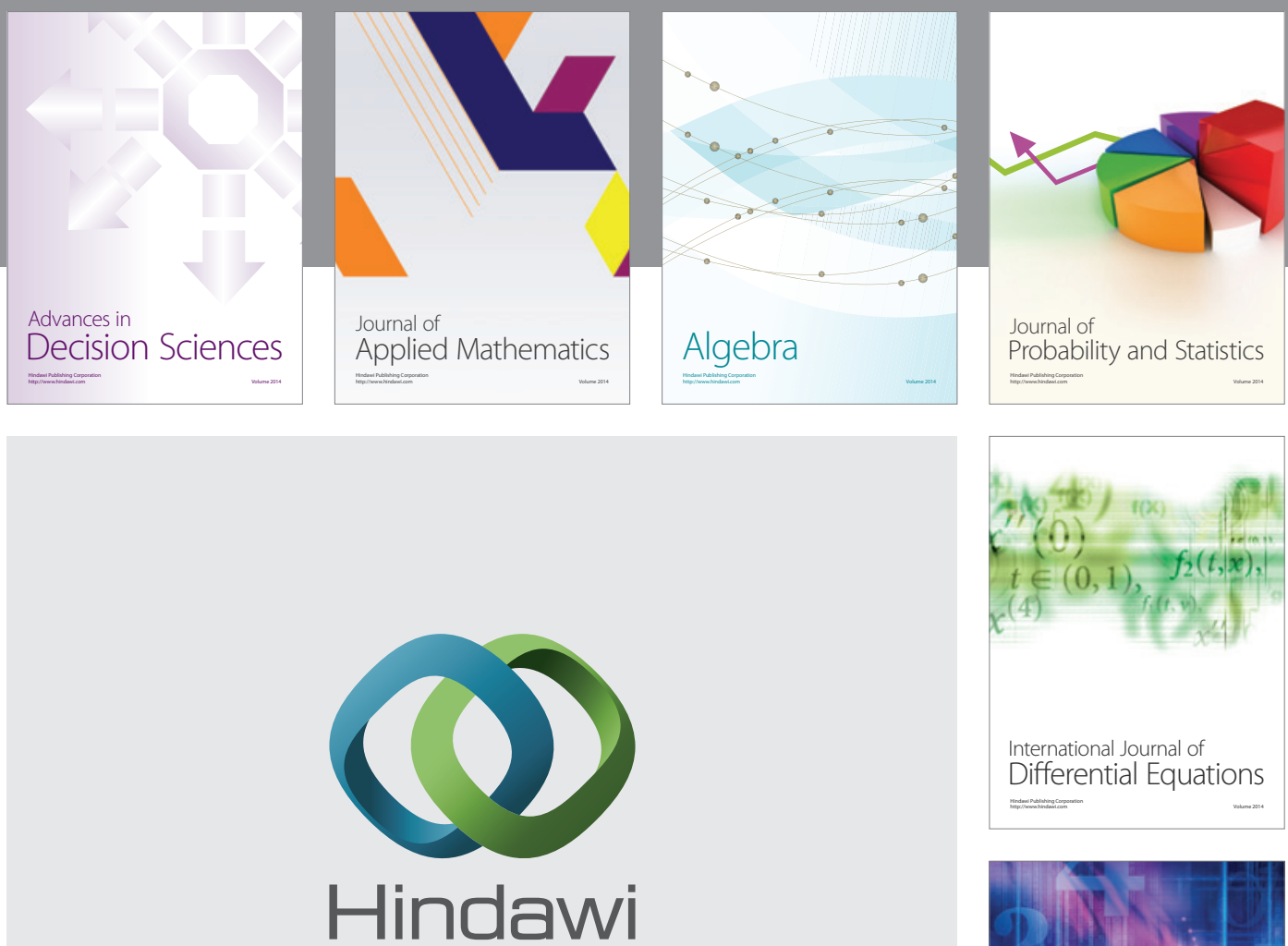

Submit your manuscripts at http://www.hindawi.com
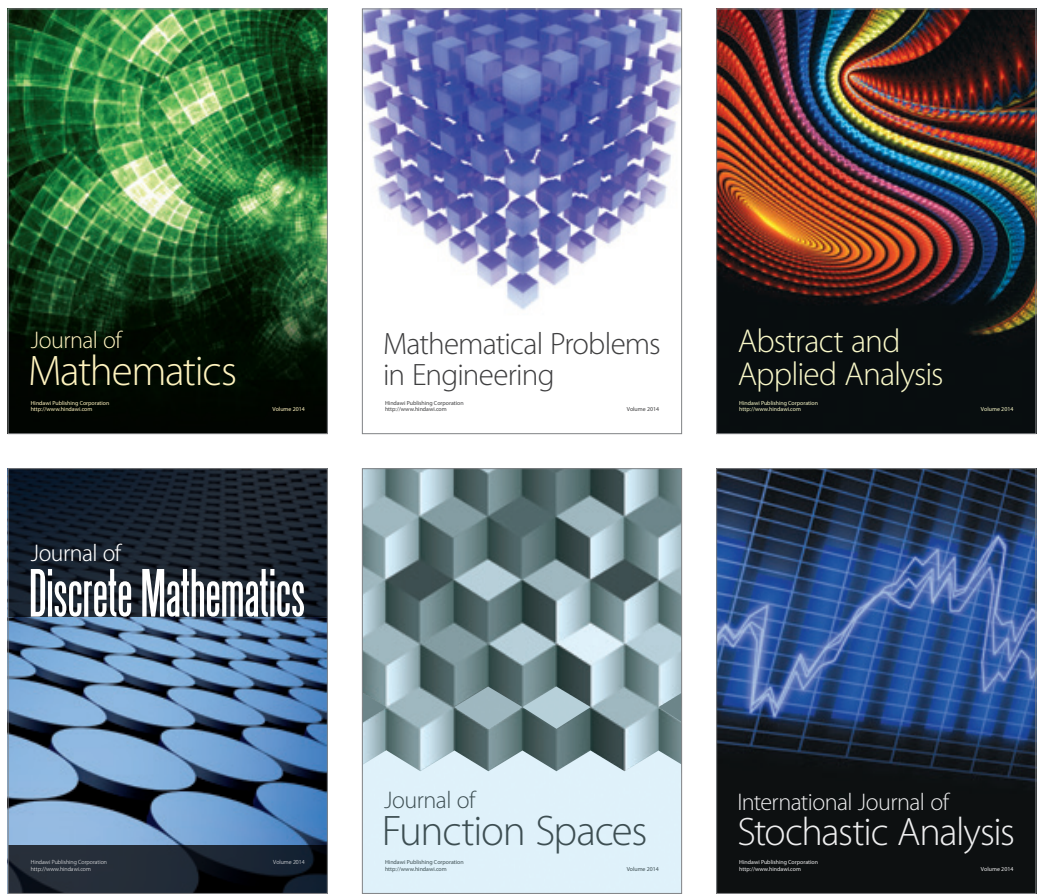

Journal of

Function Spaces

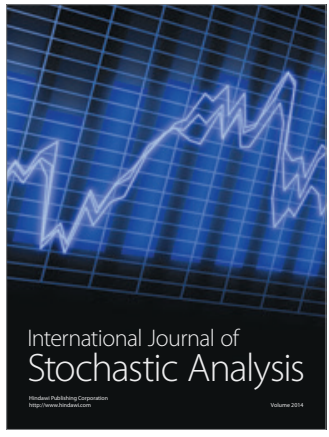

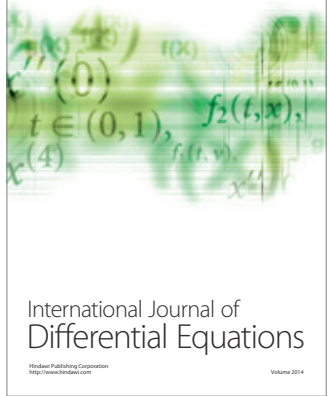
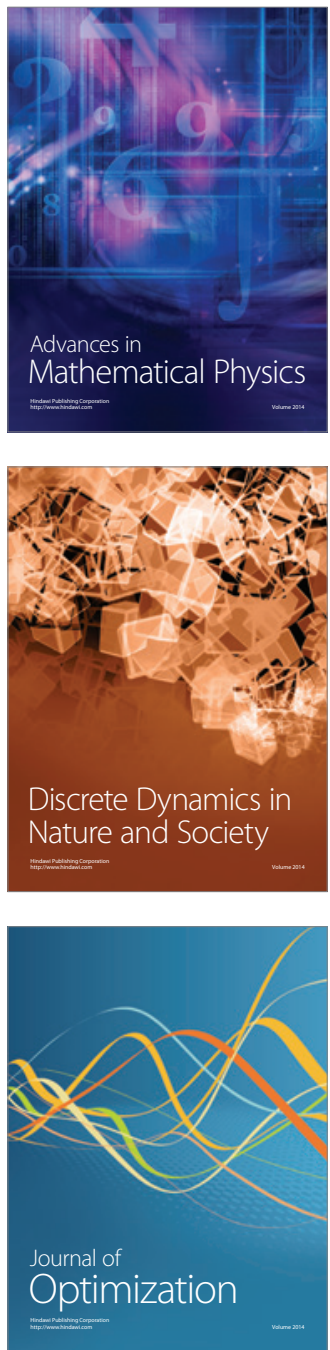